\title{
Visual similarity effects on short-term memory for order: The case of verbally labeled pictorial stimuli
}

\author{
MARIe Poirier \\ City University, London, England \\ JeAn SAINT-Aubin \\ Université de Moncton, Moncton, New Brunswick, Canada \\ AND \\ Karen Musselwhite, Thulasi Mohanadas, and Ghuson Mahammed \\ City University, London, England
}

\begin{abstract}
Four experiments examined the effect of visual similarity on immediate memory for order. Experiments 1 and 2 used easily nameable line drawings. Following a sequential presentation in either silent or suppression conditions, participants were presented with the drawings in a new, random order and were required to remember their original serial position. In Experiment 3, participants first learned to associate a verbal label with an abstract matrix pattern. Then they completed an immediate memory task in which they had to name the matrices aloud during presentation. At recall, the task required remembering either the order of the matrices or the order of their names. In Experiment 4, participants learned to associate nonword labels with schematic line drawings of faces; the phonemic similarity of the verbal labels was also manipulated. All four experiments indicate that the representations supporting performance comprise both verbal and visual features. The results are consistent with a multiattribute encoding view.
\end{abstract}

In his seminal paper, Crowder (1979) argued that similarity was an "all-purpose memory tool" and went on to discuss how similarity manipulations had a long and rich history in memory research. More recent examples of the importance of similarity - and of its counterpart, distinctiveness - in memory research abound (see, e.g., Hunt \& Worthen, 2006, for a review, as well as Avons \& Mason, 1999, and Jones, Farrand, Stuart, \& Morris, 1995, for relevant examples). Similarity and distinctiveness have been central to memory research and theorizing mainly because similarity manipulations provide a window into the nature of the representations that are the stuff of memory. Simply put, if similarity along a given dimension has an effect on memory performance, information about this dimension must somehow be represented in the memory system.

This article is concerned with the nature of the representations that support immediate memory. More specifically, the objective of the reported experiments was to investigate the role of visual characteristics in the processing of stimuli that can be verbally labeled. Hence, we set out to determine if a reliable visual similarity effect could be obtained in an immediate memory task when the to-beremembered visual items were also verbally encoded.

One of the benchmark effects in this area is that memory over the short term is influenced by phonemic similar- ity (Baddeley, 1966; V. Coltheart, 1993; Conrad \& Hull, 1964; Crowder, 1979; Poirier \& Saint-Aubin, 1996). For example, the recall of a list of rhyming words is worse than the recall of a list containing words that do not rhyme. The classic interpretation of this finding is that in order for similarity along the phonemic dimension to have an effect, the representations called upon must maintain some of the phonemic characteristics of the to-be-recalled items.

Importantly, on the basis of this effect, many of the better established models of short-term memory assume that phonemic information dominates verbal representations in short-term memory tasks (Baddeley, 1986; Baddeley \& Hitch, 1974; Nairne, 1988, 1990; Neath \& Nairne, 1995). Whether or not parallel visual similarity effects can be demonstrated is important, because such effects would indicate that the nature of the representations supporting verbal short-term memory are more complex or general than previously thought (Frick, 1985).

From a theoretical viewpoint, a number of proposals predict that visual similarity should have an effect on the immediate recall of verbal material presented in the visual modality. In the typical immediate memory task, participants are asked to recall verbatim a short list of words immediately after their presentation. More elaborate or richer encoding is usually considered a predictor

M. Poirier, m.poirier@city.ac.uk 
of enhanced memory performance. Schiano and Watkins (1981) reported evidence that is consistent with this suggestion in an experiment in which they compared immediate serial recall performance for printed words and nameable pictures in a number of conditions. For both types of materials, the results showed a phonemic similarity effect, a word-length effect, and an articulatory suppression effect, all of which are compatible with the assumption that phonemic coding is supporting performance. However, they also reported an advantage for the recall of the nameable pictures, a fact that suggests that visual information has been encoded and is playing a role in performance.

According to Hitch, Woodin, and Baker (1989), the working memory model (Baddeley, 1986; Baddeley \& Hitch, 1974 ) is another prominent proposal that suggests that visual similarity effects should be observed in standard serial recall settings. Because visually presented items are thought to be encoded in a short-term visuospatial store before an articulatory control process translates them into a phonemic code, Hitch et al. (1989) argued that evidence of visual coding should be observed under the right circumstances. This would occur, for instance, when phonological codes cannot play their usual role; in the context of the working memory model, this is assumed to be the case when tobe-remembered words are presented under an articulatory suppression requirement (the latter involves the continuous repetition of an irrelevant word or syllable and is usually considered to hamper phonemic coding [Murray, 1967]). Moreover, in discussing the same model, Logie, Della Sala, Wynn, and Baddeley (2000) noted that performance in immediate serial recall does not fall to zero under conditions of articulatory suppression; they suggested the possibility that visual codes might be supporting performance.

Finally, there are a number of proposals that have suggested that multiple features are encoded into the memory representations that support immediate memory for verbal materials, including semantic, contextual, phonemic, and visual features (Avons, 1998; Avons \& Mason, 1999; Haarmann \& Usher, 2001; Lewandowsky \& Murdock, 1989; Nairne, 1990; Neath, Brown, McCormack, Chater, \& Freeman, 2006; Neath \& Nairne, 1995; Parmentier, Tremblay, \& Jones, 2004; Tremblay, Macken, \& Jones, 2001). How much influence the latter are thought to exert on performance depends on the specifics of the model considered. However, generally speaking, on the basis of this multiattribute view, the prediction is that there should be a number of circumstances in which the influence of visual similarity on memory for verbally encoded materials can be observed.

Although there appears to be some agreement with respect to the expectation of visual similarity effects in the immediate recall of verbal materials, clear supportive evidence is sparse (see Logie, 1995, and Logie et al., 2000, for reviews). For example, Baddeley (1966) conducted one of the early studies investigating visual similarity effects in immediate serial recall and did not find reliable effects. However, as Logie et al. pointed out, he used auditory presentation. Frick (1985) examined immediate serial recall of letters with sequential and simultaneous visual presentation while requiring articulatory suppression. Vi- sual confusion errors were found to be significantly above chance for simultaneous presentation only.

A few recent studies have also found evidence of visual similarity effects in verbal short-term serial recall. These include studies with children (Hitch, Halliday, Schaafstal, and Schraagen, 1988; Hitch et al., 1989) and a study with adults that was published by Logie et al. (2000).

In the Hitch et al. (1988) study, 5-year-old and 11-yearold children completed an immediate serial recall task involving visually similar or distinct line drawings not unlike the ones used in the first two studies reported here (see the Appendix). Their results showed a visual similarity effect in the case of the young children, but not in the case of the older group. Hitch et al. (1989) confirmed these results. They also reported evidence that was interpreted as indicating that older children relied on both visual and phonemic coding: When the older children were required to perform articulatory suppression-presumably interfering with phonemic coding - a visual similarity effect was found. As was the case in the Frick (1985) article, this made it difficult to firmly conclude that visual characteristics of stimuli were encoded in silent conditions. Nevertheless, the pattern of results of these studies was consistent with the idea of multicode representations. However, as Avons and Mason (1999) have noted, interpretation of the Hitch et al. (1988; Hitch et al. 1989) studies is complicated by the fact that spatiotemporal cues were available at recall; that is, the spatial position of items varied systematically with their temporal order. Experimenters pointed to the spatial position to elicit responses, implying cues were spatial. Hence, in both the Frick (1985) and the Hitch et al. (1988; Hitch et al. 1989) studies, there was possibly some form of spatial information that could mediate the reported visual similarity effect.

Logie et al. (2000) argued that there was little evidence to suggest that the type of visual coding used by children recalling picture names might also be involved in verbal recall in adults. These authors hence set out to reexamine the question of visual similarity effects in the serial recall of verbal sequences and attempted to maximize the chances of observing the said visual similarity effects. In their first two experiments, the to-be-recalled items were words. Visual similarity was manipulated by varying the number of letters the words had in common while phonemic similarity was held constant. For example, the visually similar set for their Experiment 1 comprised the words FLY, PLY, CRY, DRY, THY, and SHY, whereas the visually distinct set comprised the words GUY, THAI, SIGH, LIE, $P I$, and RYE. Hence, all the words were phonemically similar, but the first set was more visually similar. Logie et al. also manipulated articulatory suppression; when required, suppression was carried out throughout presentation and recall. The results of both studies showed a clear visual similarity effect in that visually similar lists were more difficult to recall. Moreover, there were no interactions with articulatory suppression, suggesting that the visual similarity effect is not the result of a shift in strategiesthat is, the results of participants using phonemic coding in the silent condition and more visually based representations under suppression. However, one could argue that 
phonological coding was already disrupted by the use of a high degree of phonemic similarity (rhyming one-syllable words). As a consequence, articulatory suppression would not have produced a shift from a reliance on phonemic to visual coding. It remains to be seen whether or not the same results could be observed when reliance on phonemic coding is likely to be an efficient strategy.

In the last two experiments, Logie et al. (2000) called upon letters to test for visual similarity. They presented lists of four letters that could vary in identity or case (e.g., $\mathrm{KzQm}$ ). Participants were required to recall the items as well as their case; that is, they wrote down the letters as they appeared, including case. Visual similarity was manipulated by using set of letters with different visual characteristics. Visually similar sets consisted of letters whose upper and lower cases were similar (e.g., $K k, C c, Z z, W w$ ), whereas visually distinct sets consisted of letters with visually dissimilar case information (e.g., $T t, G g, H h, Q q$ ). Articulatory suppression was also manipulated in these experiments. In both cases, there were significant effects of visual similarity. Interactions with suppression were significant, but visual similarity effects were obtained in silent conditions.

Given the importance of these effects, it seemed appropriate to attempt to replicate and extend the finding of a visual similarity effect in the ordered recall of a sequence of verbally encoded items. The series of experiments reported by Logie et al. (2000) manipulated visual similarity under silent or articulatory suppression conditions. Moreover, in all cases there was a high degree of phonemic similarity between the stimuli to be distinguished. In the first experiment reported here, we manipulated visual similarity without employing items that were phonemically similar. In effect, we borrowed from Hitch et al. (1989) and examined the serial recall of easily nameable line drawings. However, in the study that will be reported shortly, all items were presented in the same spatial location, and there were no spatial cues to support recall. We called upon an immediate reconstruction-of-order task in which, following the presentation, participants are provided with the to-be-recalled items in a new random order. Their task is to indicate the original order in which the stimuli appeared. Using a reconstruction of order task makes sense since it has often been demonstrated that factors such as phonemic similarity have their effect on order memory (Saint-Aubin \& Poirier, 1999). Because there is the possibility that visual similarity effects will appear only when phonemic coding is made less useful, we also manipulated articulatory suppression.

\section{EXPERIMENT 1}

\section{Method}

Participants. Thirty-two participants volunteered to take part in the experiment; they were either 1st year psychology undergraduates or working within the university. They were awarded course credit or $£ 5$ for their time.

Materials. The experimental materials comprised six sets of six line drawings of everyday objects (36 drawings in total; see the Appendix for the complete list of items). Among these, there were three sets of visually similar pictures and three sets of visually distinct pictures. As shown in the Appendix, one visually similar set contained items that had similar round outlines. The items in the second similar set all had elongated shapes, and they were presented in the same oblique orientation. The third set of visually similar items contained square line drawings with similar outlines. Each of these similar sets had a control counterpart consisting of visually distinct pictures. All the drawings were easily nameable. Moreover, pairs of similar and control item sets were selected so that their names were matched for Thorndike-Lorge written word frequency, concreteness, and imageability using the MRC psycholinguistic database (M. Coltheart, 1981). Because of the constraints associated with manipulating visual similarity and controlling for word frequency, the words in the visually distinct sets were either equal to or slightly less frequent than those in the similar set (note that this favored results in the opposite direction to our main prediction, which was better recall of visually distinct items).

In this experiment, there were two blocks of 24 trials-one presented in silent conditions and one under suppression. Similar and distinct lists were mixed within these blocks. With respect to the presentation order of the trials, for each block of 24 trials, two separate orderings were generated as follows. We randomly selected without replacement one of the six item sets (three similar and three distinct) and repeated the procedure until we obtained 6 trials. We repeated this procedure four times to produce the 24 trials of a block. With this procedure-within a block of 24 trials - all item sets had to appear once before they could be presented a second time, and so on until each set had been used four times. We then repeated the whole procedure to generate a further block of 24 trials. In all cases, the order of items within lists was randomly determined with the constraint that an item could not appear twice in the same serial position within a block of 24 trials. The two versions of the trial order were counterbalanced across participants. The same was true of the order of the suppression and silence conditions.

The stimuli were presented in the center of a standard $28-\mathrm{cm}$ monitor. Timing and presentation was computer controlled except for the initiation of each trial sequence, in which the participant had to press the enter key (see details in the Procedure section).

In order to collect participant responses, response sheets reproduced the presented stimuli in a horizontal line and in a new random order relative to the presentation order (one $8.5 \times 5.5$ sheet per response; see the example in the Appendix). To avoid the possibility that participants would encode items in terms of their positions on the response sheets, a different random order was used for each response sheet with two constraints. (1) The positions of items on the answer sheet were never the same as their presentation positions, and (2) within a block of 24 lists, an item never appeared in the same serial position on the answer sheet twice. Hence, there was a separate answer sheet for each trial, each reproducing the to-be-ordered items. Because there were two separate orders for item presentation, there were also two sets of response sheets, both constructed according to the rules just described.

Procedure. Testing began by presenting participants with all 36 line drawings to be used in the experiment. The drawings appeared individually in the center of the screen within an $11-\mathrm{cm}$ square area. Each stimulus appeared with its verbal label (set in Times New Roman font, $36 \mathrm{pt}$ ) directly below it, and participants were required to read this out loud. Once the label was read, participants could view the next picture by pressing the space bar. This procedure was used to familiarize participants with the labels associated with the line drawings and to encourage all participants to use the same labels.

Familiarization with the picture labels was followed by four practice trials. Participants first had two trials in the silent condition and two in the suppression condition. Stimulus presentation began once the participant pressed the spacebar. A visual warning signal (XXXXX) was given $1 \mathrm{sec}$ before the start of each sequence of pictures. Six images then appeared one at a time in the center of the screen. Each picture was presented for $1 \mathrm{sec}$ with a blank interstimulus interval of $250 \mathrm{msec}$. At the end of each sequence, a visual recall cue (************) appeared on the screen. Participants were then required to turn over their response sheets and 
indicate the serial order of the reproduced pictures by numbering each item on the sheet: They had to first write " 1 " under the first stimuli presented, "2" under the item presented second, and so on. Strict serial order recall was called for. If participants were unable to recall a picture in a particular position, they were asked to write the position number on the side of the response sheet (in a box provided on the left; see the Appendix). Once the response sheet had been completed, the participant turned it over and put it to one side; the next answer sheet was then available, but was face down so that the presented items could not be encoded in terms of the positions of the pictures on the answer sheet. The participant hit the space bar to proceed to the next trial.

In the silent condition, participants performed the task as described previously. In the suppression condition, participants were asked to repeat aloud the phrase "tea time" at a rate of two repetitions per second, starting when they pressed the spacebar to commence presentation of a sequence and continuing until the visual recall cue had appeared They then recalled in silence. As was mentioned previously, the experimental trials were divided into two blocks of 24 trials corresponding to the suppression conditions. Because similar and distinct lists were mixed within each block of 24 trials, each of the four experimental conditions (two similarity $\times$ two suppression) was associated with 12 trials. The entire session lasted approximately $50 \mathrm{~min}$

\section{Results and Discussion}

Performance was scored according to the usual strict serial recall criterion. Experiment 1 used a reconstruction task in which the participant had to number the stimuli on a response sheet. Hence, to be considered correct, each item had to be attributed the number corresponding to its presentation position. For every participant and condition, the probability of correct recall was computed for each serial position. One participant was eliminated prior to analysis because his performance was unacceptably poor.

The results are presented in the left panel of Figure 1. The figure shows the typical suppression effect, and there appears to be a visual similarity effect under suppression conditions. Results for the silent condition appear less clear. A three-way repeated measures ANOVA was carried out on recall scores with similar-dissimilar, suppression- no suppression, and serial position (1-6) as the withinsubjects factors. For all analyses in this article, the .05 level of significance was adopted, except for simple main effect tests, in which Dunn's correction was used (Kirk, 1982).

The ANOVA revealed a significant disruptive effect of articulatory suppression on performance $[F(1,30)=$ $\left.190.45, M S_{\mathrm{e}}=0.0892\right]$, a significant effect of visual similarity $\left[F(1,30)=10.96, M S_{\mathrm{e}}=0.0217\right]$, and a significant main effect of serial position $\left[F(5,150)=41.94, M S_{\mathrm{e}}=\right.$ $0.0236]$. There was an interaction between suppression and serial position $\left[F(5,150)=10.91, M S_{\mathrm{e}}=0.0165\right]$. This interaction is attributable to the fact that the serial position curve is flatter under silent than under suppression conditions. This is likely because of a ceiling effect in the silent condition. A second interaction just missed the statistical significance criterion and involved similarity and suppression $\left[F(1,30)=4.00, M S_{\mathrm{e}}=0.0287, p=.055\right]$. Simple main effect tests revealed that visually dissimilar items were better recalled than visually similar items under suppression $\left[F(1,60)=13.55, M S_{\mathrm{e}}=0.0042\right]$, but not in the silent recall condition $(F<1)$.

Concerning these results, perhaps the first point to note is that there is a sizable effect of suppression on recall performance. Although a number of proposals have been put forward to explain how articulatory suppression hinders performance, the modal view is that it disrupts phonemic coding (but see Jones et al., 1995, and Neath $\&$ Nairne, 1995). Thus, according to this view, the task requires verbal or at least phonemic coding. On the other hand, the observed results partly support the notion that visual coding is required in the immediate serial recall of nameable pictures by demonstrating an effect of visual similarity under conditions of articulatory suppression; fewer visually similar than dissimilar items were recalled under suppression. However, in this experiment, there was no convincing evidence of a visual similarity effect in the absence of suppression.
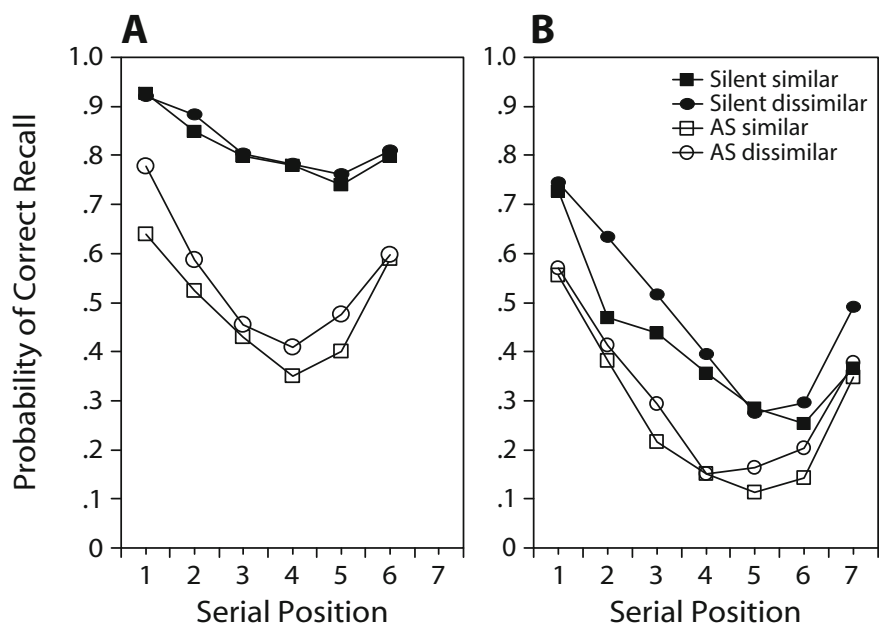

Figure 1. Probability of correct recall as a function of serial position, visual similarity, and articulatory suppression (AS). Experiment 1 results are in the left panel (A) and Experiment 2 results can be found in the right panel (B). 
These results are in line with those of Hitch et al. (1989): no visual similarity effect under the silent condition and a significant effect of visual similarity under suppression. However, there is the possibility that the absence of a visual similarity effect is attributable to a ceiling effect in the silent condition. Another possibility is that there was a mainly phonemically based encoding strategy in the silent condition and a more visually based strategy in the suppression condition, since phonemic coding is less useful in these circumstances. It remains, however, that performance in the silent condition was high and could have hindered the observation of an effect. Experiment 2 addressed this issue by reproducing Experiment 1 with longer lists.

\section{EXPERIMENT 2}

\section{Method}

Participants. Sixteen participants volunteered to take part in Experiment 2. They were all college students and were paid $\$ 7(\mathrm{Ca}-$ nadian) for their efforts.

Materials. The experimental materials were slightly different for this experiment. There were four sets of 7 items ( 28 items total), with two sets comprising visually similar items and two sets comprising visually distinct items. Because of the constraints imposed by the various controls in terms of stimulus characteristics, only two of the similar and control sets from Experiment 1 were extended to include an extra item. As in Experiment 1, picture names were equated for word length, frequency, concreteness, and imageability. Similar items had either elongated shapes (e.g., a ruler, pencil, or flute) or round shapes (e.g., a ball, button, or apple). The complete set of stimuli can be found in the Appendix.

In Experiment 2, trials were blocked according to similarity and suppression conditions $(2 \times 2)$, and each condition held 14 trials (for a total of 56 trials). Half the participants were tested with one similar and one distinct subset of items, whereas the other half were tested with the other. Hence, in this experiment, similar and distinct conditions were tested with a closed set of items - that is, the same items were presented on every trial. This more clearly emulates the conditions employed in classic studies of phonemic similarity in which closed sets of consonants were used (see, e.g., Baddeley, 1966; Conrad, 1964). The order of the four conditions produced by the combination of the similarity and suppression factors was counterbalanced across participants by using a Latin square. The order of presentation of the items within each trial was randomized for each group of 14 trials with two seven-item Latin squares. The same procedure was used to prepare the answer sheets. As before, care was taken to ensure that items were not in the same positions on the answer sheet as they were during the presentation.

Procedure. The procedure here was identical to the one used in Experiment 1, with a few exceptions. During familiarization, participants were presented with three repetitions, in a random order, of the 14 stimuli ( 7 similar and 7 distinct) that would be used in the course of the experiment for them and had to read the accompanying verbal labels aloud. This was followed by a picture naming test, in which all the stimuli were presented twice - again in random order-but without their verbal labels. Participants had to name the presented line drawing to ensure that they were familiar with the proper labels. All participants completed this task without any difficulty. The order reconstruction task began once familiarization was completed.

\section{Results and Discussion}

As occurred in the previous experiment, performance was scored according to a strict serial recall criterion. The results are presented in the right panel of Figure 1. An examination of the figure shows the usual serial position curve along with the expected decrease in performance under suppression. Also, all conditions show a lower recall level than that in Experiment 1. Most importantly, the figure reveals a lower recall performance for similar items under both quiet and articulatory suppression conditions. A $2 \times 2 \times 7$ repeated measures ANOVA confirmed these trends with main effects of visual similarity $[F(1,15)=$ $\left.8.19, M S_{\mathrm{e}}=0.0361\right]$, articulatory suppression $[F(1,15)=$ $\left.21.40, M S_{\mathrm{e}}=0.1257\right]$, and serial position $[F(6,90)=$ $\left.32.65, M S_{\mathrm{e}}=0.0484\right]$. Neither the interaction between similarity and suppression $(F<1)$ nor any of the other interactions reached significance. This experiment provides evidence of visual coding in both the silent and suppression conditions.

However, one potential criticism of the two previous experiments is that it is not clear to what degree phonemic coding is involved in performance. After all, line drawings are presented and the ordering of the same line drawings is required at recall. The fact that articulatory suppression has its typical effect on performance has very often been considered as an indication that verbal encoding is relied upon. Nonetheless, this is not the only interpretation of the effect of articulatory suppression (see, e.g., Neath \& Nairne, 1995); hence, it is perhaps not as convincing as one would hope. In Experiment 3, we adopted a different procedure in order to address this issue as well as a few others.

\section{EXPERIMENT 3}

Experiment 3 used stimuli for which a more precise measure of similarity could be computed. Abstract block matrix patterns were adapted from stimuli that were used by previous investigators (Avons \& Mason, 1999; see the Appendix). Moreover, participants were taught to associate each abstract block matrix pattern with a nonword. Hence, we avoided the constraints associated with using natural language through the selection of a pool of statistically equated nonwords. Also, to encourage phonemic encoding, participants were required to say the name of each to-be-recalled stimulus out loud during the presentation of each memory list. A further feature of this study was the manipulation of response modality. This was achieved as follows. At the point of recall, reconstruction was attempted on the basis of either a sequence of visual images or their learned verbal labels. These different response modalities were introduced as a further means of ensuring that the representations on which performance was based involved phonemic components. If visual similarity effects can be demonstrated under these conditions, then an interpretation suggesting that these items are not verbally or phonemically encoded appears more difficult to maintain.

\section{Method}

Participants. A total of 24 participants volunteered to participate in the experiment. All were undergraduate students; they either were awarded course credit or freely volunteered their time.

Materials. The experimental stimuli comprised two sets of six computer-generated graphics depicting abstract block matrix patterns ( 12 patterns in total). The patterns used were $6 \times 6$ cell matrix 
patterns with $50 \%$ of the cells filled (see the Appendix). They were displayed as black patterns on a white background, and the size of each cell was $6 \times 6 \mathrm{~mm}$. One set comprised block matrix patterns that were visually similar. This was achieved by using a prototype pattern (previously used by Avons \& Mason, 1999, Experiment 1 see their Figure 1) and changing the position of one cell for each image, with the constraints that no two patterns had the same cell changed and that the number of filled cells remained constant. This created six visually similar block matrix patterns that had 16 out of 36 cells in common. The second set of stimuli comprised six visually distinct block matrix patterns. These were created by changing the position of an average of 6.67 cells of the prototype pattern to create each pattern of the series. The six visually distinct patterns shared an average of 9.49 cells (range: $7-12$ ).

The nonwords (one for each block matrix pattern) comprised two sets of six one-syllable nonwords selected from a low-frequency item pool used by Roodenrys and Hinton (2002). Nonwords in each list were equated by the number of letters, the number of phonemes, neighborhood frequency, and biphone frequency. Low-frequency items were selected since they appeared less word-like than highfrequency items and thus benefited from having fewer phonologically similar characteristics in common with real words. To ensure that the nonwords within one visually similar list were not inadvertently more or less easy to learn or recall than the nonwords in the corresponding control list, half the participants (12) learned to associate List 1 with the visually similar patterns and List 2 with the visually distinct patterns. For the remaining 12 participants, the pattern-nonword combination was reversed.

This experiment used 18 different six-item sequences for the visually similar block matrix patterns and 18 different sequences of the six visually distinct patterns. The order of stimuli within each sequence was determined using Latin squares, with the constraint that no two sequences corresponding to each visual category had the same order of patterns. Both groups of lists were then combined such that the order of trials was alternated and predictable: No two consecutive trials held items from the same similarity category (i.e., a visually similar sequence was followed by a visually distinct sequence and vice versa). This created a series of 36 six-item sequences starting with a sequence of visually similar images, followed by a sequence of visually dissimilar images, and so on. The first four sixitem sequences (two visually similar sequences, two visually distinct sequences) were used in a practice block. To manipulate response modality, the first block of eight trials for half of the participants was followed by the ordered reconstruction of nonword (verbal) labels. The second block of eight trials was followed by the ordered reconstruction of the visually presented images. This order was repeated for the remaining 16 sequences. For the remaining participants, the first block of eight trials was followed by the ordered reconstruction of the visual presented images, and the second block of eight trials was followed by the ordered reconstruction of the verbal labels. Again, this order was repeated for the remaining 16 sequences.

Participants used response sheets that reproduced the presented items (either in their visual or in their verbal form). The order of the stimuli on the response sheets was determined using Latin squares, taking care to avoid reproducing the presentation order of the items. The stimuli were presented on a standard 28-cm PC monitor; presentation of the items and their timing were computer controlled.

Procedure. Participants were tested individually in a quiet room. Testing began with a procedure designed to familiarize the participants with the 12 images and to teach them the verbal label associated with each matrix pattern. Each image was presented in the center of a white screen within a square of $3.6 \times 3.6 \mathrm{~cm}$. Images were accompanied by their nonword labels, positioned directly below (Arial font, bold, $44 \mathrm{pt}$ ), and participants were required to read the labels out loud. The first learning trial began by presenting Visually Similar Images 1-3 individually with their corresponding verbal labels. Once each image had been studied and the label had been repeated, the participants could proceed to the next image by pressing the spacebar. The images were then presented individu- ally and without their verbal labels in a new random order, and the participants were required to provide each verbal label. Upon pressing the spacebar, the participants were provided with the image's verbal label. Images $1-3$, with and without their verbal labels, were presented in this manner for a total of six presentations each before the practice moved to Visually Distinct Images $1-3$. This procedure was continued until all images/labels had been presented on six occasions. The whole process was again repeated, but was expanded to include all six images corresponding to each visual category (e.g., Images 1-6 and 7-12 were presented together). During the learning phase, a complete cycle involved presenting each image/label a total of 144 times. This cycle was repeated until the experimenter was satisfied that the correct verbal label was consistently and easily associated with its corresponding visual image. An average of 3.58 complete practice cycles (ranging from two to six cycles) were needed for the participants to learn to associate each visual image with its verbal label. Pilot testing showed that this procedure resulted in very few naming errors during the actual memory test, with participants making two or three naming mistakes during the memory test that involved naming 216 items ( 36 lists $\times 6$ items).

Once the participants had learned to associate each image with its verbal label, four practice memory trials were given. Participants were given two trials in the visually distinct condition and two trials in the visually similar condition. Presentation began after the participant pressed the spacebar. A (***) visual warning cue was then given $1 \mathrm{sec}$ before the onset of each sequence of images. Each image was presented at the center of the screen for a duration of $1 \mathrm{sec}$, with a time lag of $250 \mathrm{msec}$ between images. Participants were required to name the image out loud as it appeared on screen. At the end of each sequence, a visual cue (******) appeared in the center of the screen. This was the participant's cue to turn to the response sheet and number the images or verbal labels from 1 to 6 in accordance with the order in which they had been presented on screen. Strict serial order recall instructions were followed; that is, the first item presented had to be the first item numbered, and so on. Participants hit the space bar to initiate the next trial. Eight trials were associated with each condition ( 2 [visual similarity] $\times 2$ [response modality]), for a total of 32 trials. The entire session lasted between 60 and $90 \mathrm{~min}$.

\section{Results and Discussion}

Responses were scored according to the usual serial recall criterion. Each item had to be ordered/numbered adequately to be considered correct. The probability of correct recall as a function of serial position, similarity, and response modality (ordering verbal labels or matrix patterns at the point of recall) is presented in Figure 2. An examination of this figure shows a disruptive effect of visual similarity, with poorer primacy performance for visually similar items than for visually distinct items. The graph displayed also appears to indicate that reconstruction performance was somewhat better with verbal labels than with visual images. A threeway repeated measures ANOVA was carried out on the recall scores with similarity (similar-distinct), response type (images-words), and serial position as factors.

Results of the ANOVA revealed a significant main effect of visual similarity $\left[F(1,23)=44.00, M S_{\mathrm{e}}=0.030\right]$, a significant effect of response modality $[F(1,23)=12.41$, $\left.M S_{\mathrm{e}}=0.024\right]$, and a significant effect of serial position $\left[F(5,115)=30.85, M S_{\mathrm{e}}=0.039\right]$. The analysis revealed one significant interaction between similarity and serial position $\left[F(5,115)=3.42, M S_{\mathrm{e}}=0.022\right]$. No other interaction reached significance. Simple main effect tests indicated that the effect of similarity was significant for the first three serial positions. 


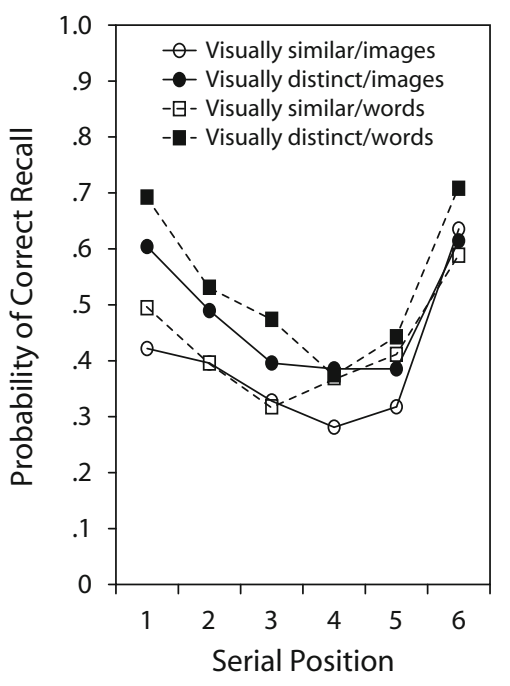

Figure 2. Experiment 3: Probability of correct recall as a function of serial position, visual similarity, and response modality (verbal labels or matrices).

The observed results provide evidence that visual coding is called for during the immediate serial ordered recall of nameable block matrix patterns, demonstrating an effect of visual similarity; fewer visually similar items were recalled regardless of response modality. In addition, the results are compatible with the notion that phonemic encoding was used by demonstrating the presence of a response modality effect; recall was superior when verbal labels rather than visual images were presented at test.

\section{EXPERIMENT 4}

The results of Experiment 3 extend the findings of the first two experiments to a situation in which the tobe-recalled items were named aloud and either visual or verbal identifiers were called upon at recall. The most important result of the previous experiment is arguably that a visual similarity effect was obtained even when stimuli were named aloud during presentation and verbal labels were used for the reconstruction response. However, given that previous research has not easily demonstrated visual similarity effects with verbally encoded items, a further demonstration with a different type of stimuli and manipulation would help to establish the reliability of the findings. This was the objective of Experiment 4. We used an alternative, more direct, and explicit way of demonstrating that verbal encoding was involved through a manipulation of phonemic similarity. We reasoned that if a phonemic similarity effect could be obtained for stimuli that also showed a visual similarity effect, this would provide further and stronger evidence supporting the idea that both visual and verbal features were playing a role in recall.

In Experiment 4, instead of block matrix patterns, we used simple line drawings depicting schematic faces (a less abstract set of stimuli). Nonword verbal labels, or "names," were again used in addition to a learning procedure.

\section{Method}

Participants. Thirty-two participants took part in this experiment. All were undergraduate students; they were awarded either course credit or a small food snack for volunteering to participate.

Materials. The experimental materials comprised two sets of line drawings depicting schematic faces (see the Appendix). One set contained seven visually similar line drawings, whereas the other set held seven visually distinct line drawings. The visually distinct set consisted of "faces" that differed in head shapes (triangle, heart, rectangle, oval, hexagon, diamond, and star). For the visually similar set, the shape of the head was always round; the eyes and nose remained the same throughout all seven stimuli, whereas the eyebrows and mouth differed. Hence, all the drawings in the similar set had three features in common. For the visually distinct set, the eyes remained the same throughout all seven stimuli, whereas the eyebrows, nose, and mouth varied from one drawing to the next. The head sizes and the number of features were kept constant across all stimuli; no color was added to the drawings.

The experimental materials also included four sets of seven nonwords. Two sets were phonemically similar, and two sets were phonemically dissimilar. The nonwords were obtained from the ARC nonword database (Rastle, Harrington, \& Coltheart, 2002). Items in each list were equated in terms of number of letters, neighborhood frequency, summed frequency of neighbors, size of phonological neighborhoods, and number of phonemes. Low-frequency items were selected to avoid any strong word-likeness effects. The combination of visually similar and distinct stimuli with the phonemically similar and distinct nonwords created four sets of stimuli. The order of stimuli within trials was determined with the use of Latin squares so that each stimulus appeared equally often in each serial position.

Stimulus presentation was computer controlled, and images of the drawings were presented on a standard $28-\mathrm{cm}$ monitor.

In order to collect participant responses, response sheets reproduced the presented stimuli in a row and in a new random order, as occurred in the previous experiments. The order of stimuli within a response set was determined using Latin squares, again so each stimulus appeared equally often in each position. However, care was taken to ensure that the order of presentation was not reproduced in the stimulus order on the response sheet.

Procedure. Phonemic similarity was manipulated between participants, whereas visual similarity was manipulated within participants. The experiment consisted of four phases. Phases 1, 2, and 3 involved learning the names associated with each visual stimulus, whereas Phase 4 involved the immediate reconstruction of the order test. In Phase 1, the stimuli appeared individually in the center of the screen. Each drawing appeared with its nonword label (set in Times New Roman font, 72 pt) directly above it, and participants were required to read this out loud. In Phase 2 of this experiment, the stimuli again appeared individually in the center of the screen. Participants tried to say the name of the schematic face out loud; after $2 \mathrm{sec}$, the label associated with the face appeared. If the participant had not done so already, she or he was to name the drawing out loud. This phase consisted of four repetitions of the two sets of seven stimuli. A Latin square was used to vary the order of presentation of the items. Phase 3 was like Phase 2 in that participants had to name each drawing aloud; however, there was only a 1-sec delay before the label appeared. This phase consisted of three repetitions of the stimulus set. Pretesting had established that this learning sequence was sufficient to virtually eliminate naming errors. The final phase involved the reconstruction of order test. Participants were presented with the face characters without the label and had to name each stimulus out loud. Stimulus presentation began once the participant pressed the spacebar. A visual warning signal (XXXXX) was displayed for $1 \mathrm{sec}$, followed by a sequence of seven schematic face drawings. The seven images appeared one at a time in the center of the screen at a rhythm of one item every $2 \mathrm{sec}$. At the end of each sequence of seven stimuli, a visual recall signal (************) indicated the start of the response phase for that trial. Participants were then required to turn to their response sheets (as before, there was a different one 
Table 1

Average Performance As a Function of Visual and Phonemic Similarity Conditions

\begin{tabular}{cccccc}
\hline & \multicolumn{2}{c}{ Visually Similar } & & \multicolumn{2}{c}{ Visually Distinct } \\
\cline { 2 - 3 } \cline { 5 - 6 } Condition & $M$ & $S D$ & & $M$ & $S D$ \\
\hline Phonemically similar & 0.49 & 0.15 & & 0.59 & 0.18 \\
Phonemically distinct & 0.59 & 0.12 & & 0.74 & 0.09 \\
\hline
\end{tabular}

for every trial) and indicate the serial order of the reproduced face characters by numbering each item in the order in which it appeared during presentation. Once the response sheet had been completed, the participants hit a key to proceed to the next trial. There were two blocks of 14 trials, one per visual similarity condition. The order of conditions as well as the association of label set to stimulus set was counterbalanced across participants. The entire session lasted approximately $50 \mathrm{~min}$.

\section{Results and Discussion}

Performance was scored according to the usual strict serial criterion. Since an order reconstruction task was used, each item had to be correctly numbered to be considered correct. Mean performance in each condition is presented in Table 1, whereas performance in each condition as a function of serial position is presented in Figure 3. Both visual similarity and phonemic similarity appear to have a detrimental effect on performance.

The data were analyzed using a mixed three-way ANOVA, with two within-subjects factors-visual similarity and serial position - and one between-subjects factor, phonemic similarity.

The ANOVA revealed a significant main effect of visual similarity $\left[F(1,30)=22.91, M S_{\mathrm{e}}=1.75\right]$, a significant main effect of group or phonemic similarity $\left[F(1,30)=9.33, M S_{\mathrm{e}}=0.25\right]$, and a significant effect of serial position $\left[F(6,180)=20.03, M S_{\mathrm{e}}=0.46\right]$. The analysis revealed one interaction that nearly reached significance, between phonemic similarity and serial position $\left[F(6,180)=3.46, M S_{\mathrm{e}}=0.08, p=.051\right]$. This ap-

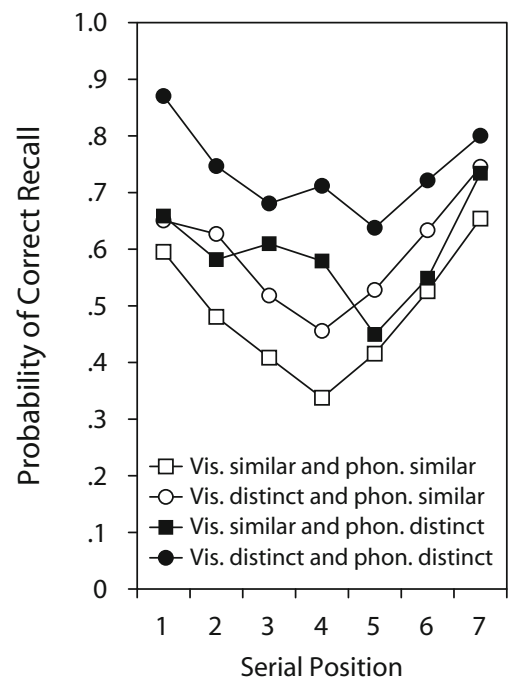

Figure 3. Experiment 4: Probability of correct recall as a function of serial position, visual similarity, and phonemic similarity. pears attributable to a larger phonemic similarity effect for Positions 3 and 4. No other interaction approached significance (all $F \mathrm{~s}<1$ ).

The present experiment has shown a clear effect of visual and phonemic similarity on short-term serial memory for nameable drawings. The effect of phonemic similarity indicates that subjects relied on a representation that maintained some of the phonemic information in the stimulus names. The observed results also provide further evidence that visual coding was called upon in this experiment.

\section{GENERAL DISCUSSION}

The aim of the experiments reported here was to determine if evidence of visual coding could be provided when the task required the short-term maintenance of a sequence of items that could be encoded verbally. In Experiment 1, the order reconstruction of nameable pictures was disrupted by visual similarity when concurrent suppression was required, but not in silent conditions; it was thought that the latter effect was attributable to a ceiling effect in the silent condition. In Experiment 2, longer lists of items were called upon; as expected, a visual similarity effect was obtained in both silent and suppression conditions. In Experiment 3, abstract matrix patterns were named aloud during presentation. This was followed by a reconstruction task in which either the patterns or their labels had to be reordered. A visual similarity effect was again observed in both cases - that is, whether the patterns or their labels had to be reordered. Taken together, the results of Experiments 1 and 2 indicate that visual similarity effects can be obtained with items that are nameable under both quiet and suppression conditions, even when list items do not share a high degree of phonemic similarity. Moreover, Experiment 3 demonstrated that the visual similarity effect occurs when the participant is required to name the stimuli out loud at study and is presented with either the verbal label or the visual image at test. Finally, Experiment 4 replicated the visual similarity effect for stimuli that were named out loud. Experiment 4 also showed that a visual similarity effect could be observed for stimuli that simultaneously produced a phonemic similarity effect. Taken together, the results of these experiments serve to establish that even under conditions in which verbal coding was clearly involved, a reliable visual similarity effect was observed.

The results build upon the findings of Avons and Mason (1999), those of Hitch et al. (1989), and those of Logie et al. (2000). Avons and Mason reported visual similarity effects for sequences of nonverbal stimuli. The results reported here extend these finding to sequences of verbally encoded items. Hitch et al. (1989) examined the performance of children and reported a pattern of results similar to that of Experiment 1; however, they used a presentation method that involved spatial as well as temporal elements. Our results show that a visual similarity effect is also found with adult participants in silent conditions as well as in suppression conditions. Finally, our results extend those of Logie et al. by providing evidence of visual similarity effects without high levels of phonemic similarity - that 
is, when phonemic information is still a useful basis for a distinctive encoding of to-be-recalled items.

Before discussing this visual similarity effect, we wish to comment on the response modality effect that was observed in Experiment 3. The results showed that immediate serial reconstruction performance was superior when responding involved verbal labels rather than line drawings - an effect perhaps indicating the greater familiarity with verbal processing of sequences. The important fact here is that the response modality effect was independent of visual similarity. This is important because it provides evidence that the visual similarity effect was not dependent upon the participants' abandoning a phonemic encoding strategy. The suggestion is that the visual properties of the stimuli were encoded in tandem with the phonemic properties in order to produce independent effects of similarity and response modality. This interpretation is reinforced by the results of Experiment 4, in which a phonemic similarity effect was observed for the same stimuli that produced a visual similarity effect.

Why was evidence of visual similarity found here, whereas it proved elusive in a number of other studies (Baddeley, 1966; Frick, 1985, 1988)? Procedural differences between studies could be involved. However, a few aspects of the present study are perhaps more likely to be of importance. First of all, we borrowed from Hitch et al. $(1988,1989)$ in using line drawings. Because this type of stimuli allow relatively good control over the number of distinctive features associated with each item, they may well have been important in obtaining visual similarity effects, especially in comparison with visually presented letters or words. For printed letters and words, there may also be an overlearned processing bias that increases the prominence of phonemic encoding. Another feature that may be relevant is that the task used here was a reconstruction of order task. This type of task is particularly sensitive to factors that affect order encoding (Saint-Aubin \& Poirier, 1999). As was suggested by Avons \& Mason (1999), it appears that visual similarity-like phonemic similarity - mainly affects order information recall. If this is the case, it may be more difficult to find evidence of visual similarity effects with standard serial recall tasks in which both the item and its position must be recalled (see Saint-Aubin \& Poirier for a more thorough discussion of these issues).

How, then, should the visual similarity effect observed here be interpreted? Perhaps the first point that needs to be made relates to the classic interpretation of the phonemic similarity effect: Phonemic confusability implies that phonemic properties are maintained in the memory record (Conrad, 1964). On the basis of this logic, the robust visual similarity effect reported here implies that visual properties of the stimuli were integrated into the representation, supporting performance. However, no one will be surprised by the statement that memory for the visual properties of stimuli is evidenced over the short term (Avons \& Phillips, 1987; Phillips \& Christie, 1977; Smyth, Hay, Hitch, \& Horton, 2005; Walker \& Davies, 2003; Walker, Hitch, Dewhurst, Whiteley, \& Brandimonte, 1997). The more novel aspect of the present find- ings is that evidence of visual coding was demonstrated for a sequence of verbally encoded items in conditions in which the phonemic information was clearly distinctive enough to support performance.

The results presented here are easy to account for if one assumes that many of the properties of a to-beremembered stimulus are encoded into the memory record and that under the right circumstances, similarity along any of these dimensions can hinder order recall. For example, one could suggest that a unitary episodic representation is produced at encoding that is then available for various processing modules-perhaps along the lines of the system suggested by the working memory model. The findings reported here are also compatible with the feature model of short-term verbal recall (Nairne, 1988, 1990; Neath \& Nairne, 1995). This model suggests that a multiattribute representation supports performance and accounts for classic effects such as phonemic similarity through a Luce-type choice rule.

It may well be possible to extend or apply other models to account for the reported findings, such as OSCAR (Brown, Preece, \& Hulme, 2000) and SIMPLE (Brown, Neath, \& Chater, 2002) and the Burgess and Hitch (1999) model. These models were developed to account for findings in the verbal domain and call upon context and distinctiveness to account for memory performance. In relation to this, Smyth et al. (2005) reported a visual similarity effect for visually similar faces in comparison with visually dissimilar faces. When discussing how their results related to recent models, they state: "There are elements of these models that need not be specific to the verbal domain. ... Context signals ... could be associated to other items, not just verbal ones. Jones et al. (1995) have proposed that serial order itself is the primary characteristic of short term recall ... and that similar effects of serial order can be found in the verbal and spatial domain" (p. 910). It is clear that our findings are in line with this general view; we also found serial position curves that are typical of immediate memory performance with verbal materials across all the item types and response modalities that we employed. This is also in line with the functional similarities between verbal, spatial, and visual short-term memory that have been pointed out by Jones et al. and Smyth et al. Finally, perhaps the main issue our findings highlight is the following. The phonemic similarity effect has been fundamental in the development of models of verbal short-term memory; we would suggest that the present findings are significant, since they indicate that the importance of phonemic coding in immediate memory for verbal material may have been overstated and, more specifically, may well be task dependent.

Our aim here was to provide evidence that visual similarity effects can be observed in immediate memory for materials that are easily nameable and verbally encoded. We observed a visual similarity effect for stimuli that were phonemically dissimilar, presented sequentially, and without any correlated variation in spatial characteristics. Moreover, this effect was observed in silent and suppression conditions when the stimuli had to be named out loud and when response required reordering the verbal labels 
associated with the to-be-remembered items. As with phonemic similarity, the pattern of results is most easily interpreted by suggesting that some of the visual characteristics of the to-be-recalled items are encoded into the memory record that supports performance. Hence, the idea that immediate memory for all verbal material is dominated by phonemic coding appears to be an overgeneralization. If early research on immediate memory had called more often upon simple line drawings rather than letters or short words, our models might not have insisted so heavily on phonemic information.

\section{AUTHOR NOTE}

This research was supported in part by an operating grant from the Natural Sciences and Engineering Research Council of Canada to J.S.-A. We thank Annie Roy-Charland and Jacinthe LeBlanc for their help in running subjects in Experiment 2. Correspondence concerning this article should be addressed to M. Poirier, Director, Memory Research Unit, Department of Psychology, City University, London, England, EC1V 0HB (e-mail: m.poirier@ city.ac.uk).

\section{REFERENCES}

Avons, S. E. (1998). Serial report and item recognition of novel visual patterns. British Journal of Psychology, 89, 285-308.

Avons, S. E., \& MAson, A. (1999). Effects of visual similarity on serial report and item recognition. Quarterly Journal of Experimental Psychology, 52A, 217-240.

Avons, S. E., \& Phillips, W. A. (1987). Representation of matrix patterns in long- and short-term visual memory. Acta Psychologica, $\mathbf{6 5}$, 227-246.

BADDELEY, A. D. (1966). Short-term memory for word sequences as a function of acoustic, semantic, and formal similarity. Quarterly Journal of Experimental Psychology, 18A, 362-365.

BADDELEY, A. D. (1986). Working memory. Oxford: Oxford University Press, Clarendon Press.

BADDELEY, A. D., \& Hitch, G. (1974). Working memory. In G. H. Bower (Ed.), The psychology of learning and motivation (Vol. 8, pp. 47-89). New York: Academic Press.

Brown, G. D. A., Neath, I., \& Chater, N. (2002). A ratio model of scale-invariant memory and identification. Unpublished manuscript.

Brown, G. D. A., Preece, T., \& Hulme, C. (2000). Oscillator-based memory and serial order. Psychological Review, 107, 127-181.

BuRgESS, N., \& Hitch, G. J. (1999). Memory for serial order: A network model of the phonological loop and its timing. Psychological Review, 106, 551-581.

Coltheart, M. (1981). The MRC psycholinguistic database. Quarterly Journal of Experimental Psychology, 33A, 497-505.

Coltheart, V. (1993). Effects of phonological similarity and concurrent irrelevant articulation on short-term-memory recall of repeated and novel word lists. Memory \& Cognition, 21, 539-545.

Conrad, R. (1964). Acoustic confusions in immediate memory. British Journal of Psychology, 55, 75-84.

ConRad, R., \& Hull, A. J. (1964). Information, acoustic confusion and memory span. British Journal of Psychology, 55, 429-432.

Crowder, R. G. (1979). Similarity and serial order in memory. In G. H. Bower (Ed.), The psychology of learning and motivation (Vol. 13 pp. 319-353). New York: Academic Press.

FRICK, R. W. (1985). Testing visual short-term memory: Simultaneous versus sequential presentations. Memory \& Cognition, 13, 346-356.

FrICK, R. W. (1988). Issues of representation and limited capacity in the visuo-spatial sketchpad. British Journal of Psychology, 79, 289-308.

HaArmann, H., \& Usher, M. (2001). Maintenance of semantic information in capacity-limited item short-term memory. Psychonomic Bulletin \& Review, 8, 568-578.
Hitch, G. J., Halliday, M. S., Schaafstal, A. M., \& Schraagen, J. M. C. (1988). Visual working memory in young children. Memory \& Cognition, 16, 120-132.

Hitch, G. J., Woodin, M. E., \& BAKER, S. (1989). Visual and phonological components of working memory in children. Memory \& Cognition, 17, 175-185.

Hunt, R. R., \& Worthen, J. (2006). Distinctiveness and memory. New York: Oxford University Press.

Jones, D. [M.], Farrand, P., Stuart, G., \& Morris, N. (1995). Functional equivalence of verbal and spatial information in serial shortterm memory. Journal of Experimental Psychology: Learning, Memory, \& Cognition, 21, 1008-1018.

KIRK, R. E. (1982). Experimental design: Procedures for the behavioral sciences (2nd ed.). Pacific Grove, CA: Brooks/Cole.

LeWANDOWsky, S., \& MuRdock, B. B., JR. (1989). Memory for serial order. Psychological Review, 96, 25-57.

LogIE, R. H. (1995). Visuo-spatial working memory. Hove, U.K.: Erlbaum.

Logie, R. H., Della Sala, S., Wynn, V., \& Baddeley, A. D. (2000). Visual similarity effects in immediate serial recall. Quarterly Journal of Experimental Psychology, 53A, 626-646.

MurRaY, D. J. (1967). The role of speech responses in short-term memory. Canadian Journal of Psychology, 21, 263-276.

NAIRNE, J. S. (1988). A framework for interpreting recency effects in immediate serial recall. Memory \& Cognition, 16, 343-352.

NaIRNE, J. S. (1990). A feature model of immediate memory. Memory \& Cognition, 18, 251-269.

Neath, I., Brown, G. D. A., McCormack, T., Chater, N., \& FreeMAN, R. (2006). Distinctiveness models of memory and absolute identification: Evidence for local, not global, effects. Quarterly Journal of Experimental Psychology, 59, 121-135.

Neath, I., \& Nairne, J. S. (1995). Word-length effects in immediate memory: Overwriting trace decay theory. Psychonomic Bulletin \& Review, 2, 429-441.

Parmentier, F. B. R., Tremblay, S., \& Jones, D. M. (2004). Exploring the suffix effect in serial visuospatial short-term memory. Psychonomic Bulletin \& Review, 11, 289-295.

Phillips, W. A., \& Christie, D. F. M. (1977). Components of visual memory. Quarterly Journal of Experimental Psychology, 29, 117-133.

PoIrier, M., \& SAINT-Aubin, J. (1996). Immediate serial recall, word frequency, item identity and item position. Canadian Journal of Experimental Psychology, 50, 408-412.

Rastle, K., Harrington, J., \& Coltheart, M. (2002). 358,534 nonwords: The ARC nonword database. Quarterly Journal of Experimental Psychology, 55A, 1339-1362.

Roodenrys, S., \& Hinton, M. (2002). Sublexical or lexical effects on the serial recall of nonwords. Journal of Experimental Psychology: Learning, Memory, \& Cognition, 28, 29-33.

Saint-Aubin, J., \& Poirier, M. (1999). The influence of long-term memory factors on immediate serial recall: An item and order analysis. International Journal of Psychology, 34, 347-352.

Schiano, D. J., \& WatKins, M. J. (1981). Speech-like coding of pictures in short-term memory. Memory \& Cognition, 9, 110-114.

Smyth, M. M., Hay, D. C., Hitch, G. J., \& Horton, N. J. (2005). Serial position memory in the visual-spatial domain: Reconstructing sequences of unfamiliar faces. Quarterly Journal of Experimental Psychology, 58A, 909-930.

Tremblay, S., Macken, W. J., \& Jones, D. M. (2001). The impact of broadband noise on serial memory: Changes in band-pass frequency increase disruption. Memory, 9, 323-331.

Walker, P., \& Davies, S. J. (2003). Perceptual completion and objectbased representations in short-term visual memory. Memory \& Cognition, 31, 746-760.

Walker, P., Hitch, G. J., Dewhurst, S. A., Whiteley, H. E., \& BranDIMONTE, M. A. (1997). The representation of nonstructural information in visual memory: Evidence from image combination. Memory \& Cognition, 25, 484-491. 
APPENDIX

Visually Similar and Distinct Stimuli Used in Experiments 1, 2, 3, and 4

\section{Visually Similar Stimuli}

\section{Experiment 1}

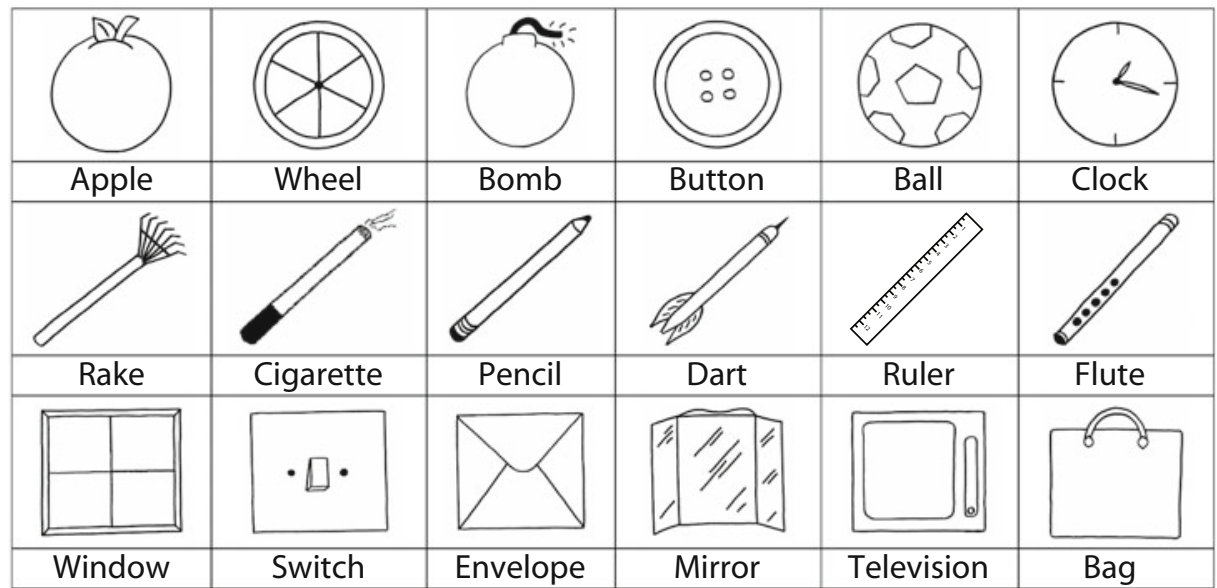

Visually Distinct Stimuli

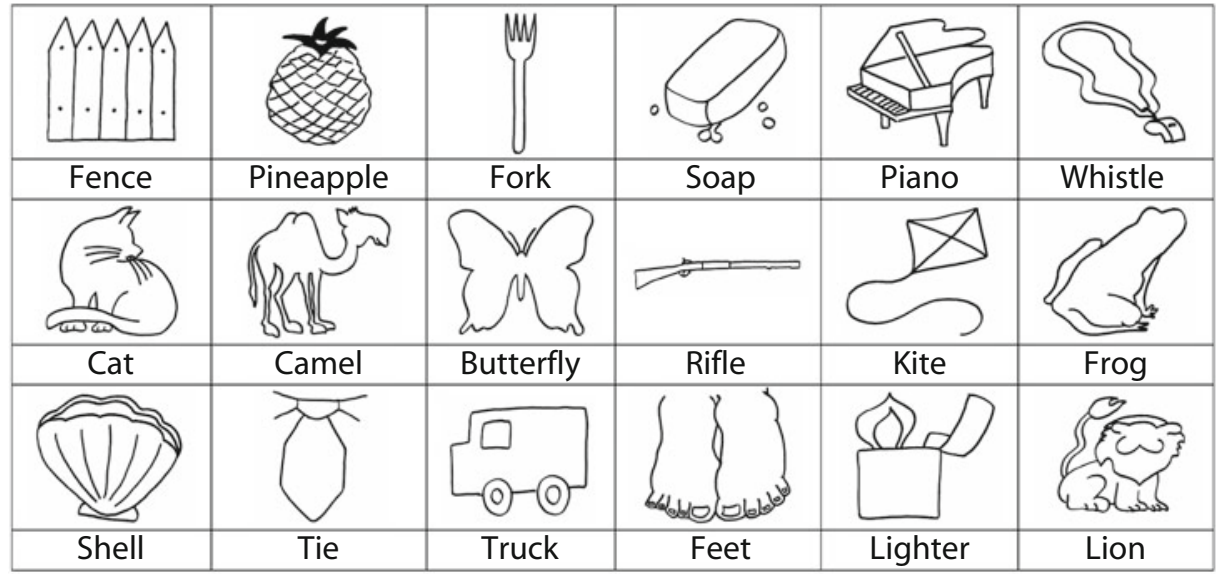

\section{Answer Sheet Example}

Participants had to number the stimuli in the order corresponding to the presentation order. This was done by writing the appropriate number in the box below the item. In the example below, the participant indicated that the apple was presented first.

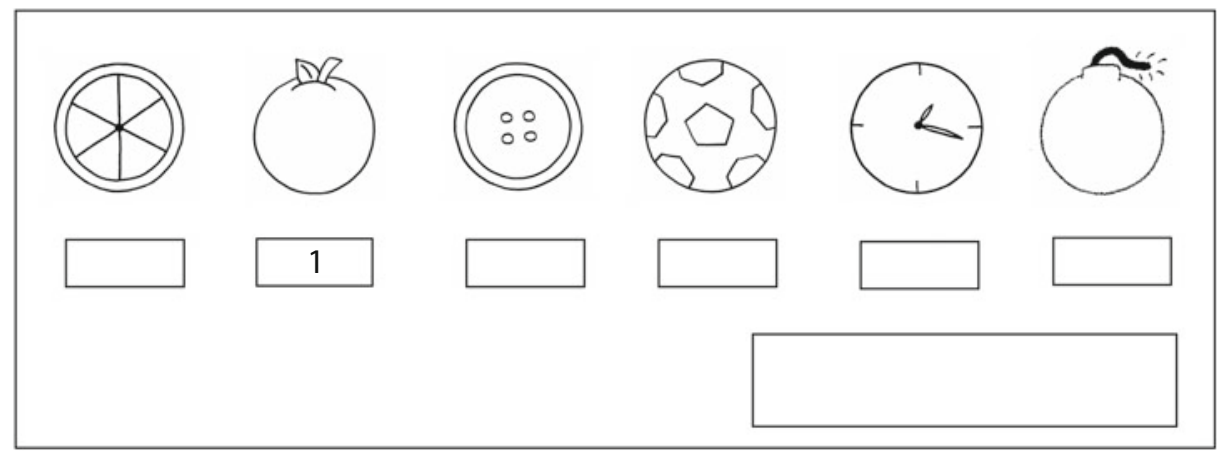




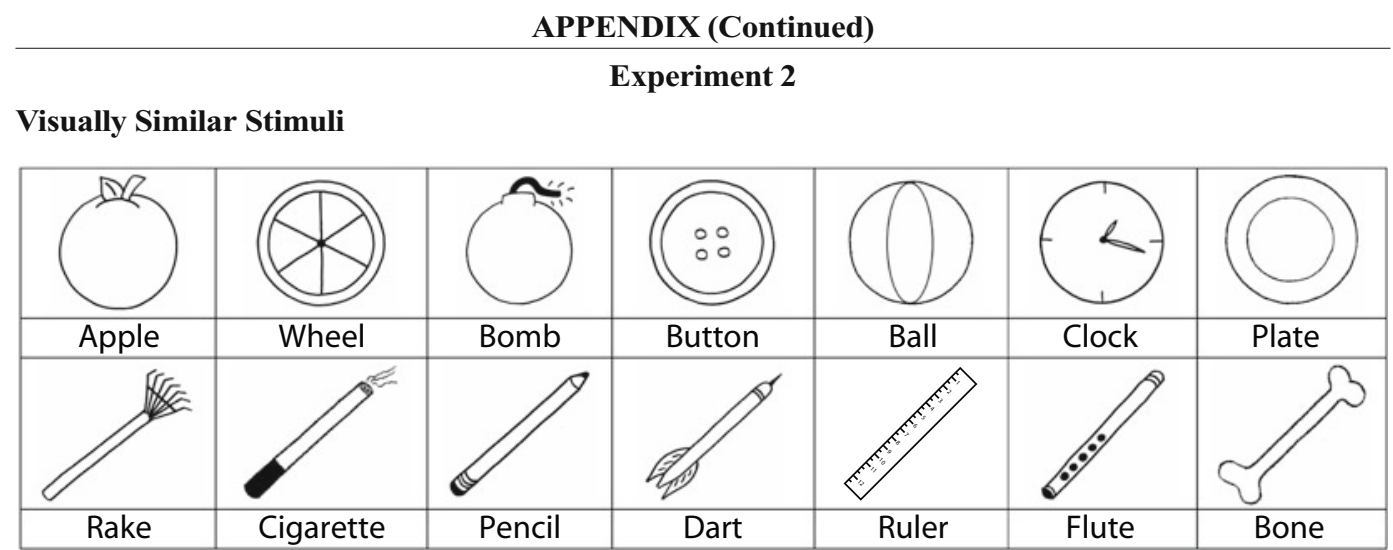

Visually Distinct Stimuli

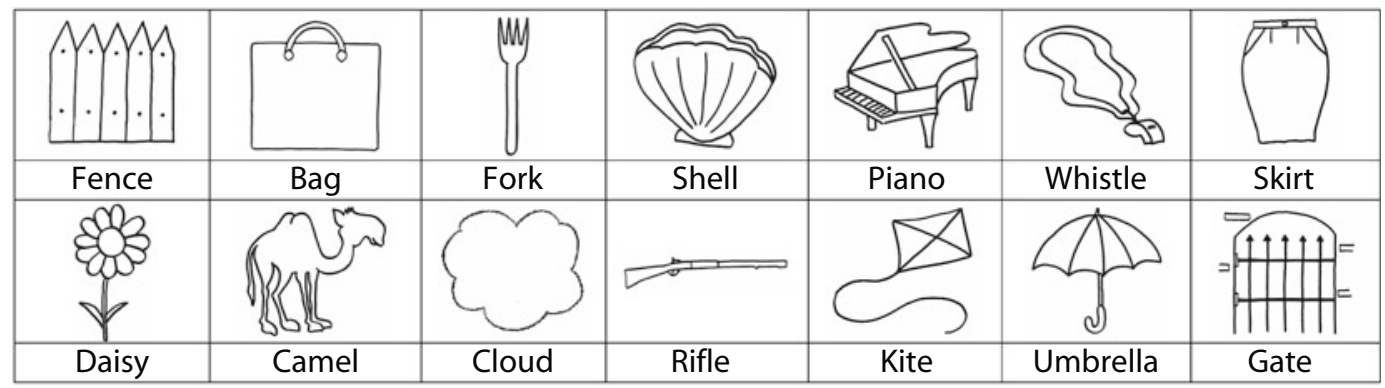

Visually Distinct Stimuli
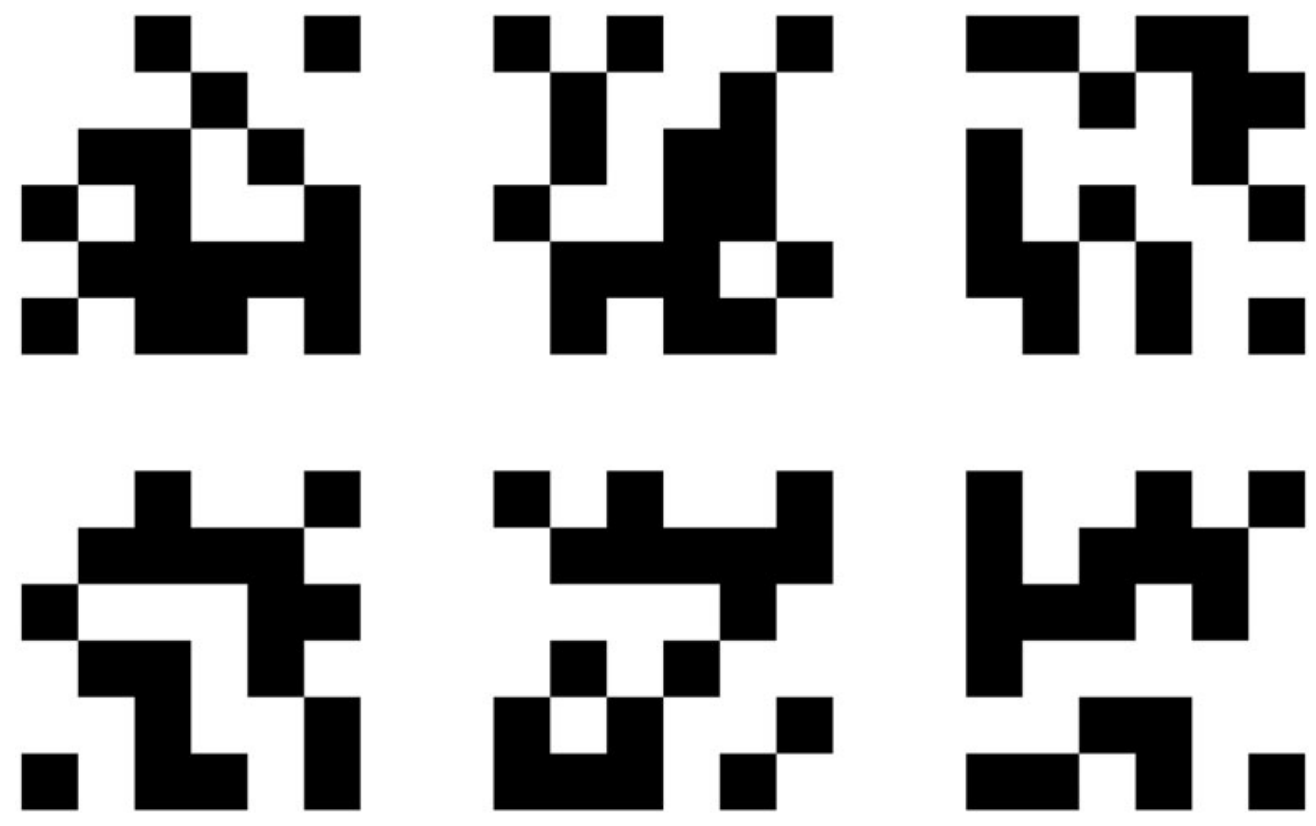


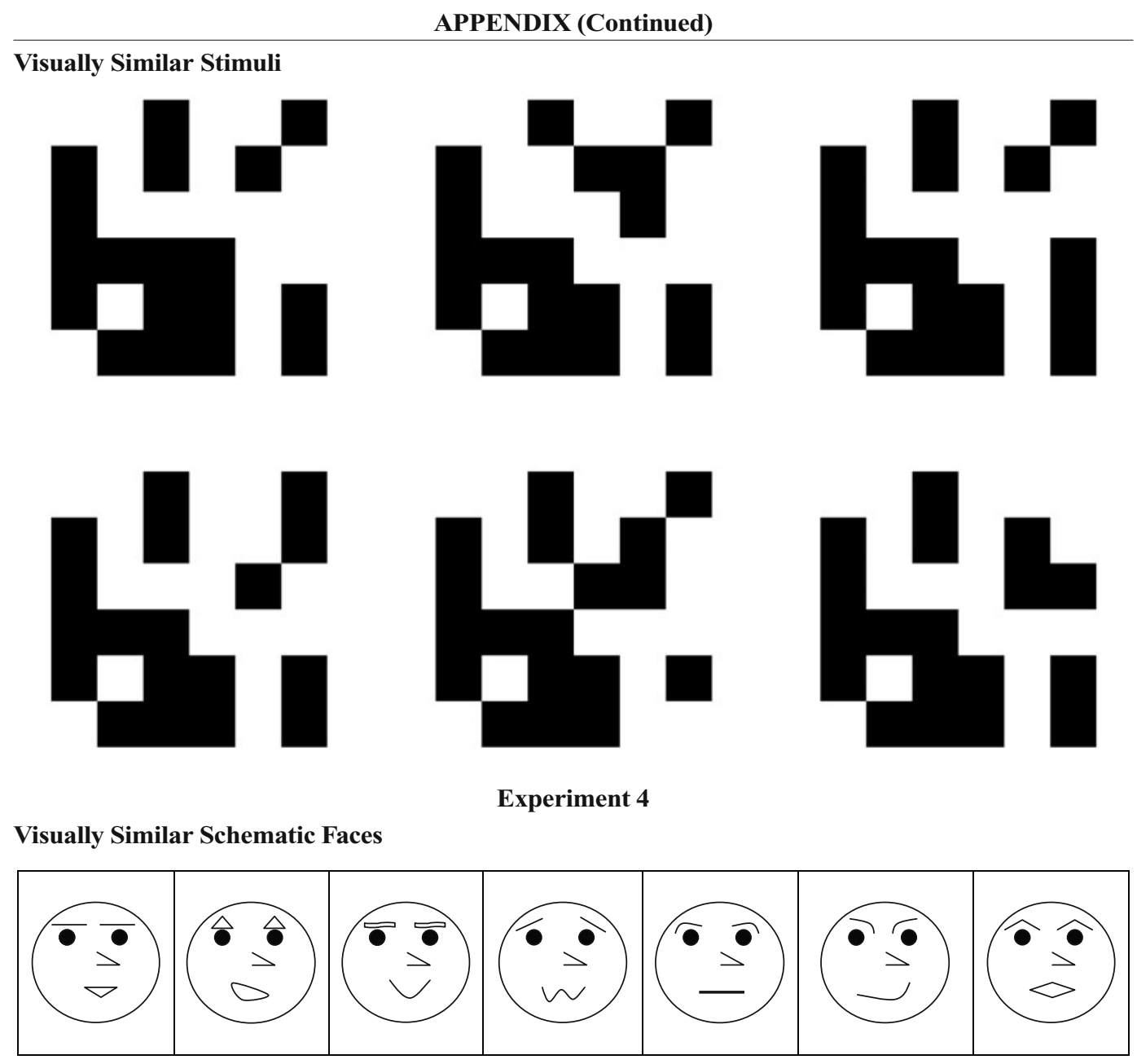

Visually Distinct Schematic Faces

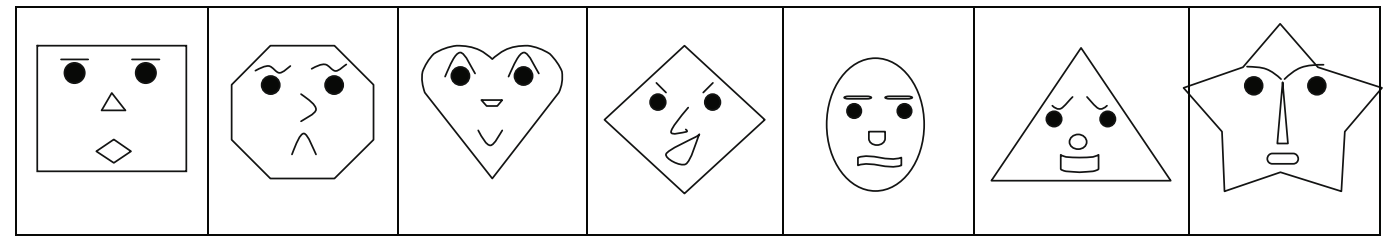

(Manuscript received April 27, 2005;

revision accepted for publication January 16, 2006.) 